\title{
T-Wave Alternans in Partial Epileptic Patients
}

\author{
Ilaria Marcantoni ${ }^{1}$, Valeria Cerquetti ${ }^{1}$, Valentina Cotechini ${ }^{1}$, Maeva Lattanzi ${ }^{1}$, Agnese Sbrollini ${ }^{1}$, \\ Micaela Morettini ${ }^{1}$, Laura Burattini ${ }^{1}$ \\ ${ }^{1}$ Università Politecnica delle Marche, Ancona, Italy
}

\begin{abstract}
Epilepsy is a chronic neurological disorder, hallmark of which is unpredictable epileptic seizures (ES). The leading cause of death in people with uncontrolled ES is the sudden unexpected death in epilepsy (SUDEP), which is believed to share genes with sudden cardiac death (SCD). Being T-wave alternans (TWA) an ECG index of $S C D$, aim of this work was to evaluate TWA occurrence in proximity of ES. Electrocardiograms (ECG) from five partial epileptic patients constituting the "Post-Ictal Heart Rate Oscillations in Partial Epilepsy" Database by Physionet were analysed for automatic TWA identification by the heart-rate adaptive match filter $(H R A M F)$. ES onsets and offsets were annotated. ECG segments starting 10min before ES and ending 10min after ES were extracted and further processed to characterize trends of median heart rate, median TWA (mTWA) and maximum TWA (MTWA) in ES proximity. Levels of $m T W A$ were significantly higher than what previously observed in a female healthy population in all ES $(46[25 ; 59] \mu V)$, pre-ES $(31[25 ; 62] \mu V)$ and post-ES $(30[26 ; 63] \mu V)$ conditions. Both mTWA and MTWA tended to increase during ES. Thus, in proximity of ES, our epileptic patients are at increased risk of SCD, possibly associated with SUDEP. Other studies defining TWA role as a biomarker for SUDEP are needed.
\end{abstract}

\section{Introduction}

Epilepsy is a central nervous system disorder in which brain activity becomes abnormal. It is recognized as one of the most common and disabling neurological disorder affecting people of all ages. Its hallmark is recurrent and unprovoked epileptic seizures (ES), whose most severe complications are damages and deaths.

A widespread cause of demise from ES is the sudden unexpected death in epilepsy (SUDEP). Every year, about $0.1 \%$ of adults and $0.02 \%$ of children suffering epilepsy die because of SUDEP. SUDEP generally comes during or in concomitance with ictus; its risk factors are young age at onset, prolonged duration of the disease, frequent
ES, low control during ES, polytheraphy with antiepileptic drugs, or lack of treatment with them. Moreover, trigger factors of SUDEP are cardiorespiratory dysregulation, systemic and cerebral circulation anomalies and hormonal or metabolic changes [1,2].

It was observed that epilepsy and ES may have important implications on cardiac function. Moreover, some cardiac function changes in the perictal and ictal moments can be considered as biomarkers and used in procedures able to forecast or identify ES. Indeed, SUDEP phenomenon is closely linked to heart function changes due to ictus [3]. Therefore, detection of the SUDEP phenomenon through the electrocardiogram (ECG) analysis in epileptic subjects may be very useful.

A common ECG marker of cardiac risk, used to forecast sudden cardiac death ( $\mathrm{SCD}$ ), is the T-wave alternans (TWA). TWA is an electrophysiological phenomenon occurring at stable heart rate (HR) characterized by a beat-to-beat alternation concerning Twave morphology, potentially involving $\mathrm{T}$-wave amplitude shape and/or polarity, in the ECG.

Still few studies have investigated TWA occurrence in proximity of ES, but those ones present in literature brought out TWA evidence in epileptic patients: Strzelczyk et al. observed that TWA rises after generalized tonic-clonic ES in patients suffering chronic uncontrolled epilepsy [2]; Sheng et al. observed that both tonic-clonic and absence ES have a very frequent TWA in association with prolonged Q-T dispersion [4]; Schomer et al. observed abnormal levels of TWA in patients with drug-resistant focal epilepsy before vagus nerve stimulation, which demonstrated to be able to reduce cardiac electrical instability (put in evidence by analysis of TWA that was used as a therapeutic target) [5]; Verrier et al. confirmed this result performing a similar study [6]. Literature suggests the need of further studies to identify the abnormal TWA incidence in patients dying for SUDEP and better define the TWA role as biomarker and therapeutic target $[5,6]$.

Aim of the present study was to evaluate TWA occurrence in proximity of ES in partial epilepsy. TWA detection and quantification may permit detection of SCD tendency, which may also imply possibility of SUDEP for epileptic patients. 


\section{Data and Methods}

\subsection{Clinical Data}

Analyzed clinical data consist in the continuous singlelead ECG signals available from the "Post-Ictal Heart Rate Oscillations in Partial Epilepsy" Database [7] in Physionet [8]. Signals were taken from five female patients ranging in age from 31 to 48 years old. They had partial ES with or without secondary generalization from frontal or temporal foci, but no clinical evidence of cardiac disease. ECG recordings were concurrently recorded with electroencephalographic recording. Recordings were made under a protocol approved by Beth Israel Deaconess Medical Center's Committee on Clinical Investigations. For some subjects more than one recording was taken and for three records 2 ES occurred for a total of 10 partial ES recorded. In Table 1 record durations and onsets and offsets of ES are reported. The latter ones were visually identified to the nearest $0.1 \mathrm{~s}$ by an experienced electroencephalographer. ECG recordings were performed at a sampling frequency of $200 \mathrm{~Hz}$ [7].

\subsection{Methods}

Data were processed in MATLAB (release R2017b). They were pass-band filtered through a bidirectional $6^{\text {th }}-$ order Butterworth filter with cut-off frequencies of $0.5 \mathrm{~Hz}$ and $40 \mathrm{~Hz}$. After that, the R peaks were detected using the Pan-Tompkins' algorithm and the baseline was removed. Baseline was computed as a cubic spline interpolation of fiducial points, placed $80 \mathrm{~ms}$ before $\mathrm{R}$ peaks.

ECG windows were extracted and preprocessed to evaluate their suitability for automatic TWA detection and quantification through the heart-rate adaptive match filter (HRAMF) method [9].

A tracing window of 40s was selected and processed every second. Then the central part of each tracing window containing 32 beats was kept to test the suitability for the actual TWA analysis by means of

Table 1. Timing of ECG recordings and ES.

\begin{tabular}{|c|c|c|c|}
\hline $\operatorname{Rec}^{1}$ & ECG dur $^{2}$ & ES onset & ES offset \\
\hline $\mathrm{R} 1$ & 1h 29' 59', & Oh 14' $36^{\prime \prime}$ & Oh 16' $12^{\prime \prime}$ \\
\hline \multirow{2}{*}{$\mathrm{R} 2$} & \multirow{2}{*}{ 3h 29' 59', } & 1h $02^{\prime} 43^{\prime \prime}$ & 1h 03'43”' \\
\hline & & $2 \mathrm{~h} 55^{\prime} 51^{\prime \prime}$ & 2h 56' $16^{\prime \prime}$ \\
\hline \multirow{2}{*}{ R3 } & \multirow{2}{*}{3 h 45' $59^{\prime}$ ' } & 1h 24' $34^{\prime \prime}$ & 1h 26' 22'” \\
\hline & & 2h 34' $27^{\prime \prime}$ & $2 \mathrm{~h} 36^{\prime} 17^{\prime \prime}$ \\
\hline $\mathrm{R} 4$ & 1h 29'59' & Oh 20' 10' & $0 \mathrm{~h} \mathrm{21'55^{ \prime \prime }}$ \\
\hline R5 & 1h 30'00', & Oh 24'07' & Oh 25' $30^{\prime \prime}$ \\
\hline \multirow{2}{*}{ R6 } & \multirow{2}{*}{ 2h 59' 59', } & $0 \mathrm{~h} 51^{\prime} 25^{\prime}$ & Oh 52' 19' \\
\hline & & $2 \mathrm{~h} 04^{\prime} 45^{\prime \prime}$ & 2h 06' 10"' \\
\hline R7 & 1h 59'59', & 1h 08'02', & 1h 09' $31^{\prime \prime}$ \\
\hline
\end{tabular}

1: record; 2: duration.
HRAMF. Detected $R$ peaks in the resized windows were used to compute the HR, the mean RR interval, the HR variability (defined as RR-interval standard deviation) and TWA frequency (TWAF, defined as half of HR). If HR variability was at most $10 \%$ of the mean RR interval and the number of beats affected by ectopic QRS or artefacts were less than $10 \%$ of total beats in each resized window, this last was classified as suitable and further analysed, otherwise it was rejected.

HRAMF works by a band-pass filter with a very narrow passing band $(0.12 \mathrm{~Hz}$-wide $)$ with the central frequency equal to TWAF. It's designed as a bidirectional $6^{\text {th }}$-order Butterworth band-pass filter. HRAMF gives in output a pseudosinusoidal signal with its maxima and minima in correspondence of ST segments or T waves if actually referring to TWA. In case, TWA amplitude is twice the pseudosinusoid amplitude, otherwise it's zero. The block diagram of the method is reported in Figure 1.

\subsection{Statistics}

ECG tracing segments that started 10min before and ended 10min after ES were extracted thank to annotations of ES onsets and offsets (Table 1). Their processing was of interest in order to characterize trends of HR and TWA in proximity of ES. Specifically, HR and TWA occurring 10-to-9 min before ES onset, 9-to-10 min after ES offset and during ES were quantified so to perform a comparison of results among different periods of time around ES happening.

Indeed, in these ECG tracing segments, the number of ECG windows (NW) that were considered suitable for TWA study were also considered. If one of the three ECG tracing segments referring to the same ES (before, after or during it) had no accepted ECG windows, the ES wasn't considered for the evaluation of the trend of variables at all. Otherwise, the median value of $\mathrm{HR}(\mathrm{mHR})$ and the median and maximum values of TWA amplitude (mTWA and MTWA, respectively) over suitable ECG windows were computed.

Given the limited number of considered ES, trends of analysed variables were evaluated in terms of $50^{\text {th }}\left[25^{\text {th }} ; 75^{\text {th }}\right]$ percentile. Comparison among results and with literature were eventually performed using the Wilcoxon rank sum test for non-normal distribution.

Statistical significance was set at 0.05 in all cases.

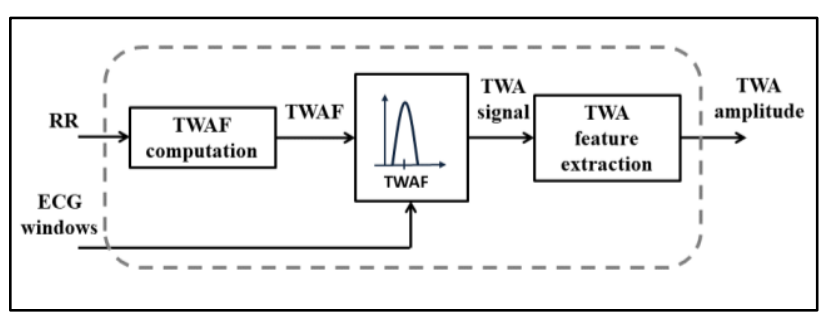

Figure 1. Block diagram of HRAMF. 


\section{Results}

Since ES characterized by even one ECG tracing segment (before, after or during them) with no suitable windows were considered not usable, the last ES was not included in the analysis because no windows were considered suitable for the TWA identification and quantification during the epileptic attack. Thus, the last record, which included only this ES, couldn't be used for the aim of this work because of the chosen criterion (described in 2.3.Statistics). Overall, TWA was analyzed in proximity of $9 \mathrm{ES}$. Results for usable ES are reported in Table 2. In it mHR (bpm), together with TWA amplitude features (mTWA, MTWA; $\mu \mathrm{V}$ ) and NW expressed in percentage are presented.

ES duration was variable, ranging from $25 \mathrm{~s}$ to $110 \mathrm{~s}$; mHR changed significantly during ES $(\mathrm{P}<0.05)$; specifically, it generally increased during their happening.

Despite the reduced number of ES, mTWA values were evaluated in comparison with values of TWA in healthy subjects present in literature. Particularly, results reported in the study of Burattini et al. [10] were taken in consideration. The comparison showed that TWA levels remained significantly $(\mathrm{P}<0.02)$ higher than what previously observed in a female healthy population, in both ES and non-ES conditions. Values of both mTWA and MTWA showed a change during ES even if not reaching statistical significance. However, they clearly showed to change during ES and to come back to the previous state after their end. This was evident considering mTWA absolute differences between values before and during ES $(17[4 ; 23] \mu \mathrm{V})$ and between values during and after ES $(16[4 ; 21] \mu \mathrm{V})$. Differences were statistically different from $0 \mu \mathrm{V} \quad\left(\mathrm{P}<10^{-4}\right)$ but not statistically different between them. Analogously, MTWA absolute differences were computed between values before and during ES $(47[26 ; 65] \mu \mathrm{V})$ and between values during and after ES $(56[17 ; 72] \mu \mathrm{V})$. Differences were statistically different from $0 \mu \mathrm{V}\left(\mathrm{P}<10^{-4}\right)$ but not statistically different between them.

\section{Discussion}

The ES-elimination criterion led to the exclusion of 1 ES and thus of an entire recording (as explained in 3.Results). Therefore, the already limited-in-number population was further reduced. This could be predictable given the difficulty to have suitable ECG windows around, but especially during ES. The most critical period to analyze TWA in epileptic patients is during ES, very particular moments in which cardiac conduction disorders are common $[3,11]$. It's for this reason that the majority of rejected windows were present during ES. Changes of mHR during ES confirmed results of several studies present in literature. Indeed, $\mathrm{mHR}$ changing reflects the state of the autonomic nervous system and epileptic patients appear to have an altered autonomic cardiac control [3,12-17].

Few studies in literature have considered repolarization anomalies, like QT dispersion or TWA incidence, in epileptic patients [4]. Some of them also provided a quantification of TWA and results obtained in this study are comparable with them, if measurement conditions are similar $[2,5,6]$.

Table 2. Trend of features extracted from ECG tracing segments in proximity and during ES.

\begin{tabular}{|c|c|c|c|c|c|c|c|c|c|c|c|c|}
\hline \multirow[b]{2}{*}{ ES } & \multicolumn{4}{|c|}{ BEFORE ES } & \multicolumn{4}{|c|}{ DURING ES } & \multicolumn{4}{|c|}{ AFTER ES } \\
\hline & $\begin{array}{l}\mathbf{m H R} \\
(\mathrm{bpm})\end{array}$ & $\begin{array}{c}\text { mTWA } \\
(\mu \mathrm{V})\end{array}$ & $\begin{array}{c}\text { MTWA } \\
(\mu \mathrm{V})\end{array}$ & $\begin{array}{l}\text { NW } \\
(\%)\end{array}$ & $\begin{array}{l}\mathbf{m H R} \\
(\mathrm{bpm})\end{array}$ & $\begin{array}{c}\text { mTWA } \\
(\mu \mathrm{V})\end{array}$ & $\begin{array}{c}\text { MTWA } \\
(\mu \mathrm{V})\end{array}$ & $\begin{array}{l}\text { NW } \\
(\%)\end{array}$ & $\begin{array}{l}\mathbf{m H R} \\
(\mathrm{bpm})\end{array}$ & $\begin{array}{c}\text { mTWA } \\
(\mu \mathrm{V})\end{array}$ & $\begin{array}{c}\text { MTWA } \\
(\mu \mathrm{V})\end{array}$ & $\begin{array}{l}\text { NW } \\
(\%)\end{array}$ \\
\hline 1 & 72 & 11 & 16 & 100 & 114 & 10 & 45 & 64 & 103 & 14 & 33 & 100 \\
\hline 2 & 67 & 63 & 83 & 10 & 86 & 46 & 105 & 100 & 59 & 30 & 66 & 100 \\
\hline 3 & 61 & 26 & 46 & 100 & 84 & 47 & 70 & 100 & 61 & 45 & 89 & 94 \\
\hline 4 & 65 & 31 & 48 & 100 & 96 & 54 & 145 & 72 & 70 & 29 & 42 & 100 \\
\hline 5 & 70 & 40 & 54 & 100 & 119 & 34 & 102 & 66 & 69 & 27 & 41 & 100 \\
\hline 6 & 60 & 25 & 40 & 100 & 98 & 27 & 194 & 9 & 71 & 23 & 35 & 100 \\
\hline 7 & 91 & 25 & 84 & 100 & 125 & 20 & 30 & 26 & 85 & 62 & 90 & 100 \\
\hline 8 & 72 & 62 & 84 & 100 & 68 & 85 & 110 & 79 & 69 & 66 & 113 & 100 \\
\hline 9 & 70 & 98 & 158 & 100 & 69 & 74 & 111 & 100 & 70 & 90 & 167 & 100 \\
\hline tot & $\begin{array}{c}70 \\
{[64 ; 72]} \\
\end{array}$ & $\begin{array}{c}31 \\
{[25 ; 62]} \\
\end{array}$ & $\begin{array}{c}54 \\
{[45 ; 84]} \\
\end{array}$ & $\begin{array}{c}100 \\
{[100 ; 100]}\end{array}$ & $\begin{array}{c}96 \\
{[81 ; 115} \\
\end{array}$ & $\begin{array}{c}46 \\
][25 ; 59] \\
\end{array}$ & $\begin{array}{c}105 \\
{[64 ; 120]} \\
\end{array}$ & $\begin{array}{c}72 \\
{[55 ; 100]} \\
\end{array}$ & $\begin{array}{c}70 \\
{[67 ; 75]} \\
\end{array}$ & $\begin{array}{c}30 \\
{[26 ; 63]} \\
\end{array}$ & $\begin{array}{c}66 \\
{[40 ; 96]} \\
\end{array}$ & $\begin{array}{c}100 \\
{[100 ; 100]}\end{array}$ \\
\hline
\end{tabular}


This work proposed a comparison with values of TWA generally considered normal in healthy subjects. For this aim one of Burattini's works was used [10]. In it, resting ECG recordings from 142 healthy subjects (77 males and 65 females), used as controls, fed HRAMF to quantify TWA. Aim of the analysis was to investigate possible differences between males and females and the conclusion was that TWA is a gender-related phenomenon; thus, TWA was reported for male and female, separately [10].

Since the population under examination in the present work was completely female, results were compared with those obtained in the 65 females treated in [10]. Considering mTWA and MTWA values, TWA was high around ES and during them; moreover, it changed during the attack. Thus, SCD risk, possibly linked to SUDEP is higher for our epileptic patients than healthy population.

In this study it is right to stress the fact that obtained results are to be considered preliminary. Indeed, the population under examination in this context was reduced to five female subjects, of which a general classification of epilepsy (partial) was provided. Thus, larger and deeper studies are needed to better define TWA role as a biomarker for SUDEP.

A study on a larger population, with female and male subjects, also to evaluate a possible difference related to gender, is certainly useful. Future studies may evaluate a possible dependence of TWA to acquisition lead [18] and a possible correlation between ES duration and TWA incidence. Longer ECG tracings should be available in order to define a trend of TWA in subjects that suffer by $\mathrm{ES}$, also including the actual basal state.

\section{Conclusion}

In proximity of ES, considered partial epileptic patients showed increased levels of TWA and thus are at increased risk of SCD, possibly associated with SUDEP. Since the population available in the database examined here was too limited, more studies are certainly desirable to identify TWA function in SUDEP prediction.

\section{References}

[1] C. E. Stafstrom, and L. Carmant, "Seizures and epilepsy: an overview for neuroscientists," Cold Spring Harb. Perspect. Med., vol. 5, no. 6, pp. 1-18, Jun. 2015.

[2] A. Strzelczyk et al., "Postictal increase in T-wave alternans after generalized tonic-clonic seizures," Epilepsia, vol. 52, no. 11, pp. 2112-2117, Nov. 2011.

[3] K. Jansen, and L. Lagae, "Cardiac changes in epilepsy," Seizure, vol. 19, no. 8, pp. 455-460, Oct. 2010.

[4] D. Sheng, and X. Cheng, "Analysis of the Q-T dispersion and $\mathrm{T}$ wave alternans in patients with epilepsy," Yangtze Medicine, vol. 1, no. 2, pp. 109-116, Jun. 2017.

[5] A. C. Schomer, B. D. Nearing, S. C. Schachter, and R. L. Verrier, "Vagus nerve stimulation reduces cardiac electrical instability assessed by quantitative T-wave alternans analysis in patients with drug-resistant focal epilepsy," Epilepsia, vol. 55, no. 12, pp. 1996-2002, Dec. 2014.

[6] R. L. Verrier, B. D. Nearing, B. Olin, P. Boon, and S. C. Schachter, "Baseline elevation and reduction in cardiac electrical instability assessed by quantitative T-wave alternans in patients with drug-resistant epilepsy treated with vagus nerve stimulation in the AspireSR E-36 trial," Epilepsy Behav., vol. 62, pp. 85-89, Sept. 2016.

[7] I. C. Al-Aweel et al., "Post-ictal heart rate oscillations in partial epilepsy," Neurology, vol. 53, no. 7, pp. 1590-1592, Oct. 1999.

[8] A. L. Goldberger et al., "PhysioBank, PhysioToolkit, and PhysioNet: components of a new research resource for complex physiologic signals," Circulation, vol. 101, no. 23, pp. E215-E220, Jun. 2000.

[9] L. Burattini, W. Zareba, and R. Burattini, "Automatic detection of microvolt $\mathrm{T}$-wave alternans in Holter recordings: effect of baseline wandering," Biomed. Signal Process. Control, vol. 1, no. 2, pp. 162-168, Apr. 2006.

[10] L. Burattini, W. Zareba, and R. Burattini, "Identification of gender-related normality regions for T-wave alternans," Ann. Noninvasive Electrocardiol., vol. 15, no. 4, pp. 328336, Oct. 2010.

[11] M. Nei, R. T. Ho, and M. R. Sperling, "EKG abnormalities during partial seizures in refractory epilepsy," Epilepsia, vol. 41, no. 5, pp. 542-548, May 2000.

[12] K. Jansen, C. Varon, S. Van Huffel, and L. Lagae, "Periictal ECG changes in childhood epilepsy: implications for detection systems," Epilepsy Behav., vol. 29, no. 1, pp. 7276, Oct. 2013.

[13] C. Opherk, J. Coromilas, and L. J. Hirsch, "Heart rate and EKG changes in 102 seizures: analysis of influencing factors," Epilepsy Res., vol. 52, no. 2, pp. 117-127, Dec. 2002.

[14] E. Suorsa et al., "Heart rate dynamics in temporal lobe epilepsy- a long-term follow-up study," Epilepsy Res., vol. 93, no. 1, pp. 80-83, Jan. 2011.

[15] T. Tomson, M. Ericson, C. Ihrman, and L. E. Lindblad, "Heart rate variability in patients with epilepsy," Epilepsy Res., vol. 30, no. 1, pp. 77-83, Mar. 1998.

[16] K. S. Eggleston, B. D. Olin, and R. S. Fisher, "Ictal tachycardia: the head-heart connection," Seizure, vol. 23, no. 7, pp. 496-505, Aug. 2014.

[17] J. Pavei et al., "Early seizure detection based on cardiac autonomic regulation dynamics," Front. Physiol., vol. 8, no. OCT, Oct. 2017, Art. No. 765.

[18] L. Burattini, S. Man, R. Burattini, and C. A. Swenne, "Comparison of standard versus orthogonal ECG leads for T-wave alternans identification," Ann. Noninvasive Electrocardiol., vol. 17, no. 2, pp. 130-140, Apr. 2012.

Address for correspondence:

Laura Burattini, $\mathrm{PhD}$

Department of Information Engineering,

Università Politecnica delle Marche,

Via Brecce Bianche 12,

60131, Ancona, Italy.

E-mail address.1.burattini@univpm.it. 